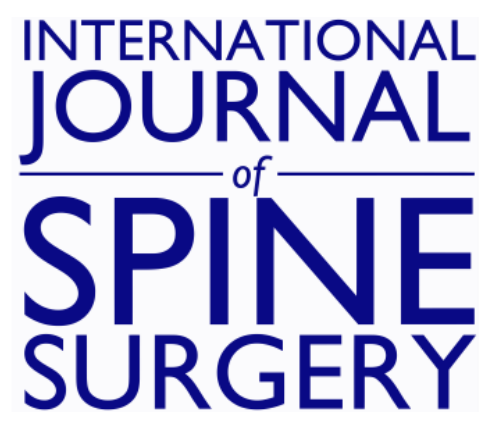

\title{
Postoperative Sagittal Spinal Alignment Changes Following Corrective Surgery in Adult Spinal Deformity
}

Ahmed Albayar, Ashwin Ramayya, Patricia Zadnik Sullivan, Jang Yoon, Comron Saifi, Abigail Roche, Zarina S. Ali, William C. Welch, Vincent Arlet and Ali K. Ozturk

Int J Spine Surg 2021, 15 (6) 1082-1089

doi: https://doi.org/10.14444/8194

http://ijssurgery.com/content/15/6/1082

This information is current as of April 26, 2023.

Email Alerts Receive free email-alerts when new articles cite this article. Sign up at:

http://ijssurgery.com/alerts

The International Journal of Spine Surgery

2397 Waterbury Circle, Suite 1,

Aurora, IL 60504, Phone: +1-630-375-1432 


\title{
Postoperative Sagittal Spinal Alignment Changes Following Corrective Surgery in Adult Spinal Deformity
}

\author{
AHMED ALBAYAR, MD $^{1}$; ASHWIN RAMAYYA, MD ${ }^{1}$; PATRICIA ZADNIK SULLIVAN, MD ${ }^{1}$; JANG YOON, MD, \\ MSC $^{1}$; COMRON SAIFI, MD ${ }^{2}$; ABIGAIL ROCHE, BS ${ }^{1}$; ZARINA S. ALI, MD ${ }^{1}$; WILLIAM C. WELCH, MD, FACS, \\ FICS $^{1}$; VINCENT ARLET, MD ${ }^{2}$; AND ALI K. OZTURK, MD ${ }^{1}$ \\ ${ }^{I}$ Department of Neurosurgery, Hospital of University of Pennsylvania, Pennsylvania, Philadelphia, USA; ${ }^{2}$ Department of Orthopedic Surgery, Hospital of University of \\ Pennsylvania, Pennsylvania, Philadelphia, USA
}

\begin{abstract}
Background: There is a paucity of literature covering the spinal alignment changes following adult spinal deformities (ASD) corrective surgeries. In theory, patients' posture and overall alignment may vary with postoperative pain, bracing, and other external variables requiring further radiographic follow-up. The purpose of the study is to investigate changes in sagittal alignment in the first 3 months postoperatively.

Methods: This is a retrospective case series of ASD patients who underwent deformity surgeries from October 2015 to June 2018. Patients < 40 years old, had < 6 levels fused, had acute proximal junctional kyphosis (PJK) or failure, or lacked imaging were excluded. Physiologic measures, spine alignment changes measured in whole-spine radiographs. Lumbar lordosis (LL), thoracic kyphosis (TK), and sagittal vertical axis (SVA) at immediate and 3-month postoperative time points were measured, then compared via 2-sample Student $t$ tests. Furthermore, TK after upper thoracic to pelvis (UT-P) fusions was compared with lower thoracic to pelvis (LT-P) fusions via paired $t$ test.

Results: Thirty-six patients ( 24 females, $67 \%$ ) with a mean age of 61.5 years (range, $40-75$ years) were included. Spinal alignment comparisons showed a significant increase in TK at the 3 -month time point $(P=0.006)$. Additionally, wide variations in SVA (range, 47-144 mm) were noted, yet not statistically significant, likely due to the changes being in both positive and negative directions $(P=0.18)$. No significant difference was found when TK was compared in the UT-P vs LT-P groups.

Conclusions: Our results suggest that as postoperative pain subsides and the body settles into its new alignment, significant changes occur in spine sagittal parameters in the subacute period following surgery.
\end{abstract}

Level of Evidence: 4.

Lumbar Spine

Keywords: adult spine deformity, flat back syndrome, subacute postoperative change, sagittal vertical axis, thoracic kyphosis

\section{INTRODUCTION}

Adult spinal deformities (ASD) are characterized by a loss of the normal curvature and alignment of the spinal column, and can lead to severe pain. Surgical correction is increasingly becoming an acceptable option in select patients to relieve pain, regain function, and improve long-term quality of life. ${ }^{1-3}$ Relatively recent improvements in spinal instrumentation and neuromonitoring, as well as our understanding of spine sagittal alignment, have made these high-risk operations reasonably safe and feasible. ${ }^{4}$ Moreover, the prevalence of ASD has been increasing in the past few decades, reaching $32 \%$ to $68 \%$ in individuals who are 65 years or older. ${ }^{5}$ The population at risk for ASD is also increasing, and by some estimates, the elderly population (individuals 60 years of age or older) is expected to reach nearly $22 \%$ of the general population of the United States in 2050. ${ }^{6}$
In an aging population, progressive degenerative disease can change the alignment of the spine leading to ASD. Spine sagittal and spinopelvic parameters include fixed anatomical considerations such as pelvic incidence, as well as modular factors such as pelvic tilt and lumbar lordosis (LL). Pelvic incidence, defined as the angle derived from a line perpendicular to the sacral endplate (at the superior border of S1) and the line defining the bifemoral axis, is limited by a patient's anatomy. For neutral anatomic alignment, the pelvic incidence should be within $10^{\circ}$ of LL (defined as the Cobb angle from the superior lumbar vertebral endplate to the inferior endplate of the lowest lumbar vertebral body). ${ }^{7,8}$ Indeed, every patient's correction must be tailored to their anatomic needs. For this reason, accurate preoperative and postoperative measurements of spinal alignment are imperative as both overcorrection and undercorrection have been shown in numerous studies to lead to worse outcomes and increase the likelihood 
of proximal junctional kyphosis (PJK) and worsening quality of life. ${ }^{9-12}$

In many cases, the goal of corrective surgery for ASD is to reduce a patient's positive sagittal balance, essentially creating a neutral position of the head over the sacrum. These corrections are planned based on preoperative radiographs and targeted surgical interventions introduce additional degrees of LL through anterior column lengthening or posterior column shortening via various osteotomies. Pedicle screws and rods are utilized to reinforce the new anatomical alignment and immediate postoperative $\mathrm{x}$-rays are taken to identify if the surgery achieved a successful correction. ${ }^{7,8}$ However, relatively little is known regarding the subacute changes in spinal alignment and biomechanics in the first few months following surgery. The timing and significance of such changes, if they occur, can affect the long-term corrective outcome of the surgical interventions and may have implications on further treatment. Surgeons will typically rely on spinal alignment measured during an immediate postoperative period to judge the degree of surgical correction achieved and will obtain further imaging at regular time-points at clinical follow-up. ${ }^{13}$ Therefore, it is critical for spine surgeons who perform deformity correction surgeries to have an accurate understanding of how spinal parameters are expected to change over time, as this may influence clinical decision-making. ${ }^{14}$

We hypothesized that pain and deconditioning immediately following surgery in these patients may yield immediate postoperative $\mathrm{x}$-rays that differ from ones obtained in the subacute period once the acute surgical pain subsides and following a course of therapy. In the present study, we sought to understand changes that may occur in spinal alignment following ASD surgery in the first 3 months compared to the initial postoperative imaging.

\section{MATERIALS AND METHODS}

\section{Patient Selection}

This case series is a single-institution, retrospective study of 36 ASD patients undergoing spinal deformity corrective surgeries by 2 spine surgeons between October 2015 and June 2018. After the hospital institutional review board's approval, patients' data were obtained from medical records. The baseline data were collected and reviewed retrospectively after deidentification by 2 different reviewers to ensure accuracy. Inclusion criteria were age $>40$ years and available whole-spine lateral erect $\mathrm{x}$-ray scans at acute postoperative (during initial hospital stay) and 3-month postoperative timepoints. All patients underwent elective adult degenerative spinal deformity correction surgeries. In each case, the determination of spine procedures, approach, and extent of fusion was based on several factors. These factors included the degree of thoracic kyphosis (TK), global balance, and the surgeon's judgment. The exclusion criteria were patients with acute PJK and proximal junctional failure (PJF) within the first 6 months, patients who did not undergo 3 -month postoperative imaging or had less than 6 spinal levels fused in surgery. Pain control protocols aimed to start patients on oral pain medications shortly after the surgery. The standard dose of Oxycodone for moderate postoperative pain is $5 \mathrm{mg}$ and $10 \mathrm{mg}$ for severe pain. Muscle relaxants such as Cyclobenzaprine or Diazepam and nonnarcotic pain medications such as Acetaminophen and Ketorolac were employed in maximized doses to aid the shift to nonnarcotic pain control.

\section{Preoperative and Intraoperative Variables}

The medical records of patients were reviewed to obtain data such as age at surgery, sex, body mass index (BMI), smoking status, length of stay (LOS), return to the operating room (OR), previous spine surgeries, proportion of staged spine surgeries, number of levels fused anteriorly and posteriorly, the utilization of hooks, osteotomies and hyperlordotic cages (HLCs), status of instrumentation failure, and estimated blood loss (EBL).

\section{Radiographic Assessment}

Immediate (during index hospital admission) and 3 to 6 months postoperative whole-spine lateral x-ray scans were obtained and deidentified for further reviewing. Surgimap spine software (Nemaris Inc, New York, NY), which employs precalculated anatomical relations between accurately identified anchor points and spinal anatomical measurements, ${ }^{15,16}$ was used to obtain the radiographic measurements for the sagittal vertical axis (SVA), TK, and LL.

\section{Calculation}

We assessed whether there were differences between TK, LL, and SVA measured immediately postoperatively and at the 3-month follow-up by applying Student $t$ tests (paired, 2 tailed). We considered a $P$ value of $<0.05$ to be statistically significant. In addition, we compared the changes in spinal alignment in 2 groups, upper thoracic to pelvis (UT-P) to the lower thoracic to 
Table. Patients' characteristics and surgical details.

\begin{tabular}{lc}
\hline Characteristics and Surgical Details & $\boldsymbol{N}=\mathbf{3 6}$ \\
\hline Gender, female patients, $n(\%)$ & $29(81 \%)$ \\
Age at surgery, y, mean (SD) & $61.5 \mathrm{y}(8.7)$ \\
BMI, kg/m ${ }^{2}$, mean (SD) & $27.7(5.6)$ \\
LOS, d, mean (SD) & $7.8(4.2)$ \\
Smoking status, active smokers, $n(\%)$ & $6(17 \%)$ \\
History of previous spine surgery, $n(\%)$ & $28(78 \%)$ \\
Staged spine procedure, $n(\%)$ & $9(25 \%)$ \\
Collective EBL, mL, mean (SD) & $1406.4(720.4)$ \\
Return to OR, $n(\%)$ & $9(25 \%)$ \\
LT-P fusions, $n(\%)$ & $25(69 \%)$ \\
UT-P fusions, $n(\%)$ & $11(31 \%)$ \\
Combined anterior and posterior spine procedures, & $32(89 \%)$ \\
$n(\%)$ & \\
Use of hooks, $n(\%)$ & $24(67 \%)$ \\
Smith-Peterson osteotomies, $n(\%)$ & $32(89 \%)$ \\
Use of HLCs, $n(\%)$ & $30(83 \%)$ \\
Vertebroplasty, $n(\%)$ & $4(11 \%)$ \\
\hline
\end{tabular}

Abbreviations: BMI, body mass index; EBL, estimated blood loss; LOS, length of stay; LT-P, lower thoracic to pelvis fusion cases; OR, operating room; SD, standard deviation; UT-P, upper thoracic to pelvis fusion cases.

${ }^{\text {ac}}$ Collective EBL refers to the sum of EBL after spine procedures either staged (anterior and posterior surgeries) or EBL of 1-stage spine surgery.

pelvis (LT-P) fusions, using Student $t$ test to investigate variabilities occurring with the different fusion lengths.

\section{RESULTS}

\section{Patient Data}

Thirty-six patients (29 females, $81 \%$ ) were included in the study (Table). The mean age at surgery was 61.5 years. The mean BMI was $27.7 \mathrm{~kg} / \mathrm{m}^{2}$ (SEM, $0.99 \mathrm{~kg}$ ) $\mathrm{m}^{2}$ ). The mean LOS was 7.8 days (SEM, 0.7). Six patients $(17 \%)$ were current smokers. Twenty-eight patients $(78 \%)$ had a history of previous spine surgeries. Nine patients $(25 \%)$ underwent staged spine surgeries. Eight patients (24\%) were reported to have instrumentation failure as a complication of the ASD surgery. The mean EBL was $1406 \mathrm{~mL}$ (SEM, 121.7 $\mathrm{mL})$. Nine patients $(25 \%)$ returned to the OR due to wound-related complications or instrumentation failure. Twenty-five patients (69\%) underwent LT-P fusion while 11 patients $(31 \%)$ underwent long UT-P fusions. In all cases, pedicle screws were placed using a freehand technique. Among this cohort, 32 (89\%) cases received anterior lumbar interbody fusion (ALIF) procedures. Nine of $11(81.8 \%)$ UT-P cases and $23(92 \%)$ of 25 LT-P cases underwent ALIF procedures. Moreover, hooks were used in $24(67 \%)$ cases and osteotomy procedures were performed in $32(89 \%)$ cases. Twelve cases underwent single-level Smith-Paterson osteotomy (SPO) while 20 cases underwent SPO at multiple levels. HLCs were used in $30(83 \%)$ cases. Finally, vertebroplasty was performed in $4(11 \%)$ cases (Table).

We found that TK (T4-T12) Cobb angles were significantly higher at the 3-month follow-up interval as compared to the immediate postoperative x-ray $(t[36]=2.91$, $P=0.006$ ) (Figures $1 \mathrm{~A}$ and 2 ). We did not find statistically significant differences in comparing the immediate and the 3-month postoperative radiographic LL and SVA measurements. However, we observed a trend toward increased LL Cobb angles $(P=0.21)$ and decreased SVA $(P=0.18)$ at 3 months postsurgery (Figure $1 \mathrm{~B}$ and $\mathrm{C}$ ). Notably, there was a wide variability in SVA with some patients with extreme positive and negative changes that may have affected the statistical analysis, thus rendering it insignificant (Figures 3 and 4).

To assess whether the increased TK at 3-month follow-up was related to the extent of the fusion construct, we compared patients that had upper thoracic (T2-4) to pelvis
A

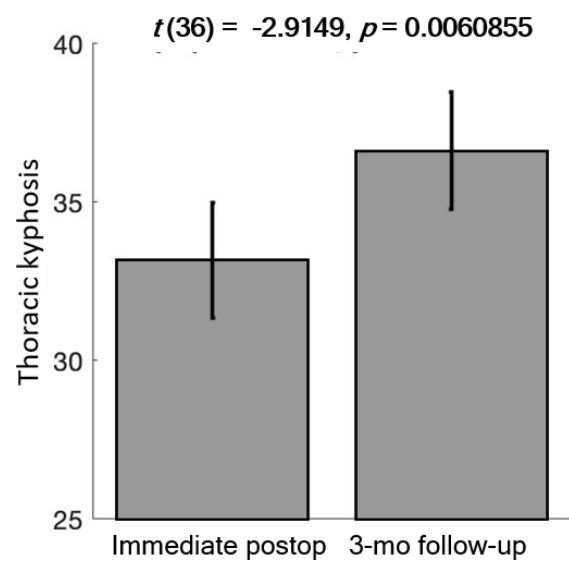

B

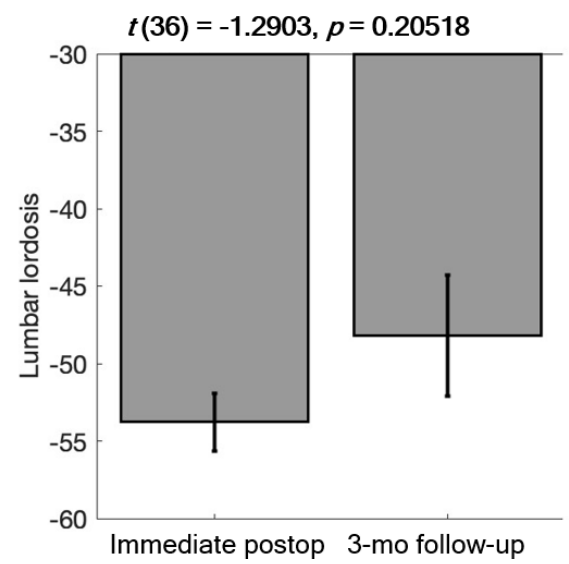

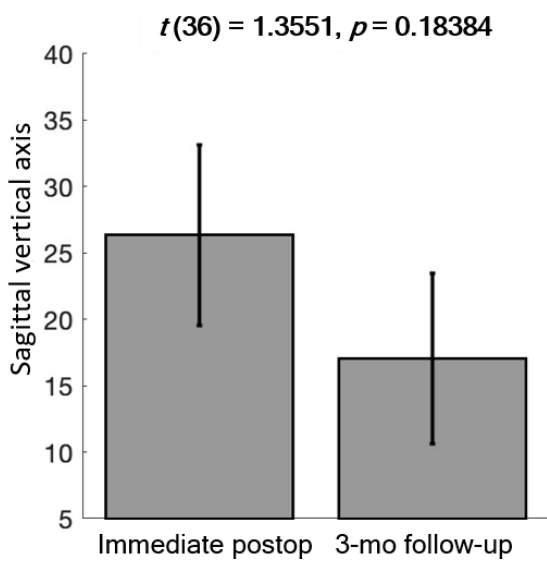

Figure 1. Bar plots representing radiographic changes at 3 months postsurgery compared to immediate postoperative radiographs. (A) Significant changes in thoracic kyphosis $(\mathrm{TK})$ Cobb angles $(t[36]=2.91, P=0.006)$. (B) Lumbar lordosis and $(\mathrm{C})$ sagittal vertical axis changes did not reach statistical significance $(P=$ 0.21 and $P=0.18$, respectively). 


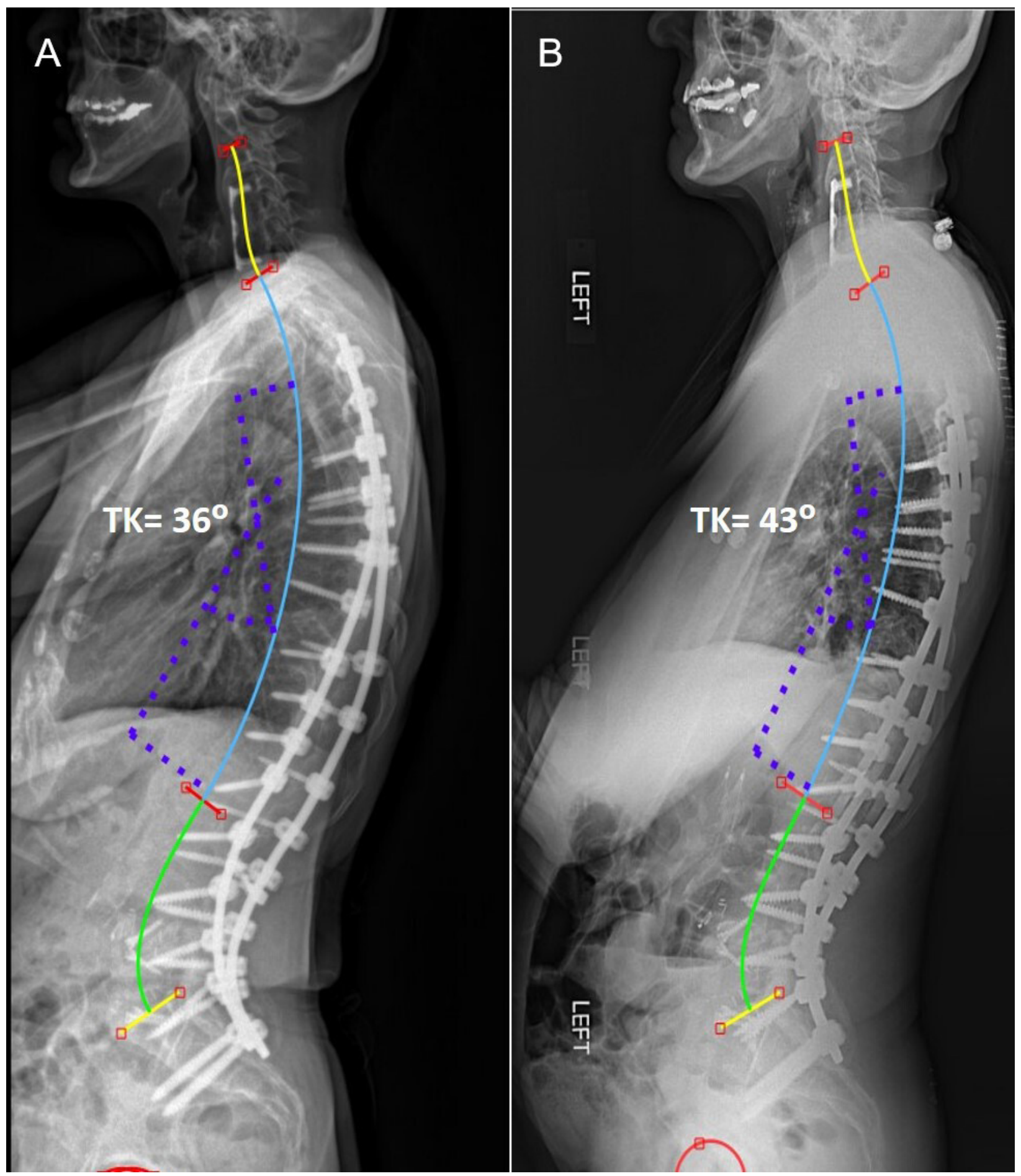

Figure 2. Lateral whole-spine radiographic scans representing changes in thoracic kyphosis (TK) at 3-month postoperative time point compared to immediate postoperative TK. (A) Immediate postoperative radiographs showing TK Cobb angle measurement, 36 ${ }^{\circ}$. (B) The 3-month postoperative radiographs showing TK Cobb angle measurement; $43^{\circ}$. The difference between TK measurements is $7^{\circ}$.

fusions vs lower thoracic (T9-11) to pelvis fusions. We did not observe a statistically significant difference between these groups $(P>0.5)$, suggesting that the increase in TK at 3 months was not related to the length of the fusion construct and occurred even with rigid instrumentation of the thoracic spine (Figure 5).

\section{DISCUSSION}

There is a lack of accurate knowledge about the expected course of postoperative changes in spinal alignment following ASD corrective surgeries. This lack may diversify practice patterns based on individual 


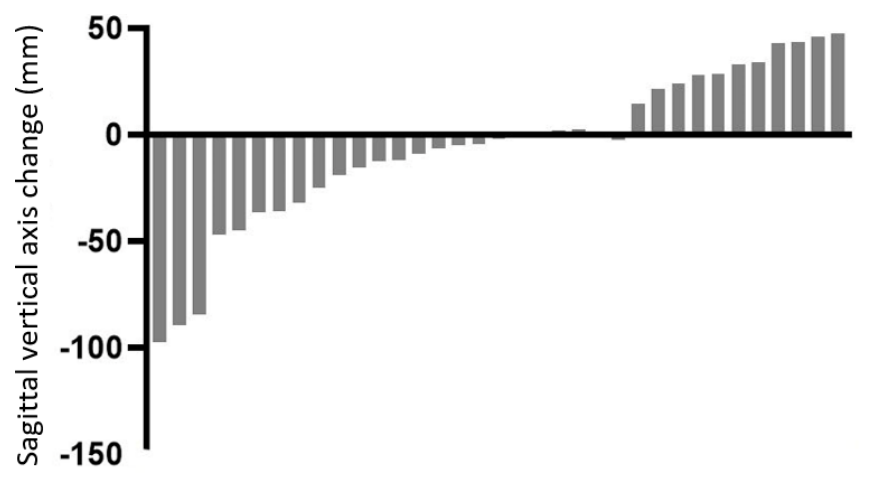

Figure 3. Graphic representation of changes in the sagittal vertical axis. Changes in the 3-month postoperative period have shown wide variability.

experiences. We hypothesized that changes in spinal alignment, additional to the immediate postoperative changes, may occur in the first few months following corrective surgery for ASD. In this study, we assessed the changes in Cobb angle measurements for TK, LL, and SVA in 36 patients who underwent ASD corrective surgery at immediate postoperative timepoint and at 3 to 6 months postoperatively.

TK Cobb angles showed a statistically significant increase at 3 months postoperatively (Figures 1A and 2 ). This could be due to a physiologic increase in TK as LL is restored, since patients with flat back syndrome or who have lumbar hypolordosis preoperatively tend to decrease their thoracic kyphoses in a compensatory effort. ${ }^{17}$ Patients with flat back syndrome and lumbar hypolordosis preoperatively tend to decrease their thoracic kyphoses in a compensatory effort to stay upright. Yasuda et al have reported a case series of 65 patients older than 50 years who underwent corrective surgeries for ASDs to evaluate the changes in thoracic curvature postoperatively. They observed a fairly consistent increase in TK postoperatively between the timepoints of their evaluation and a correlation between the changes in TK specifically and the patient's age and preoperative TK values. ${ }^{18}$ Increased TK may also be pathologic and an early indicator of impending PJK/PJF. Furthermore, this increase in TK was seen in patients instrumented to both the upper (T2-4) and lower thoracic (T9-11) spine, indicating that the presence of rigid hardware did not alter this outcome (Figure 5). Because acute PJK/PJF may take place in the first 6 months, we excluded these patients so that the results would not be confounded due to the extreme focal kyphosis seen in these cases, as we did not believe these would contribute accurately to the natural course of these patients. Furthermore, the causes of PJK/PJF are varied, and patient age, bone quality, and other factors come into play.
Notably and quite interestingly, the SVA measurements varied widely between immediate postoperative and 3 to 6 months timepoints (Figure 3). We observed both significantly positive (Figure 4) and negative changes in comparable numbers, likely explaining the lack of statistical significance (Figure 1C). We hypothesize that these changes may at least in part be related to postoperative pain and thus the immediate postoperative $\mathrm{x}$-ray may indeed be somewhat unreliable. As the pain subsides, gradual mechanical adjustments may take place as the body settles into its new alignment ${ }^{19,20}$ and subsequently, we believe, more accurate and reliable measurements can be made. Furthermore, ASDs are 3-dimensional deformities that are affected by the presence of many demographic, genetic, and geriatric factors. These factors may also cause variability in how sagittal alignment reacts to corrective surgeries. Therefore, ASD postoperative management will benefit from age-specific expectations of the postoperative radiographic changes. ${ }^{19}$

We believe our results demonstrate that significant changes occur in spinal alignment in the first few months following ASD correction surgery and that conclusions about undercorrection or overcorrection should be reserved until after the immediate postoperative period and some rehabilitation has taken place. For example, a patient with a significantly positive SVA following surgery may indeed tempt the surgeon to entertain reoperation for improved alignment, but our data suggest that this decision may best be deferred. In addition, obtaining spinal radiographic measurements at multiple timepoints. For example, immediate and 3-month postoperative time points may facilitate growing a knowledge repository of the natural course of postoperative changes. In a relevant retrospective analysis, Boddu et al have found evidence supporting the practice of routine postoperative radiographic evaluations of patients following ASD corrective surgeries. ${ }^{21}$

The limitations of this study should be considered in the context of its design as it included a small sample size in a single high-level spine deformity center which limits the generalizability of its results. In addition, we did not stratify the patients into various subgroups based on surgical approaches (anterior vs posterior, staged vs single stage, etc) and the patient-reported outcomes were not obtained as part of the analysis as it was solely focused on the postoperative progress of sagittal alignment. Lastly, while we aimed to investigate the changes in the acute and subacute period following surgery, we did not compare our measurements with longer-term follow-up imaging. However, based on our findings and 


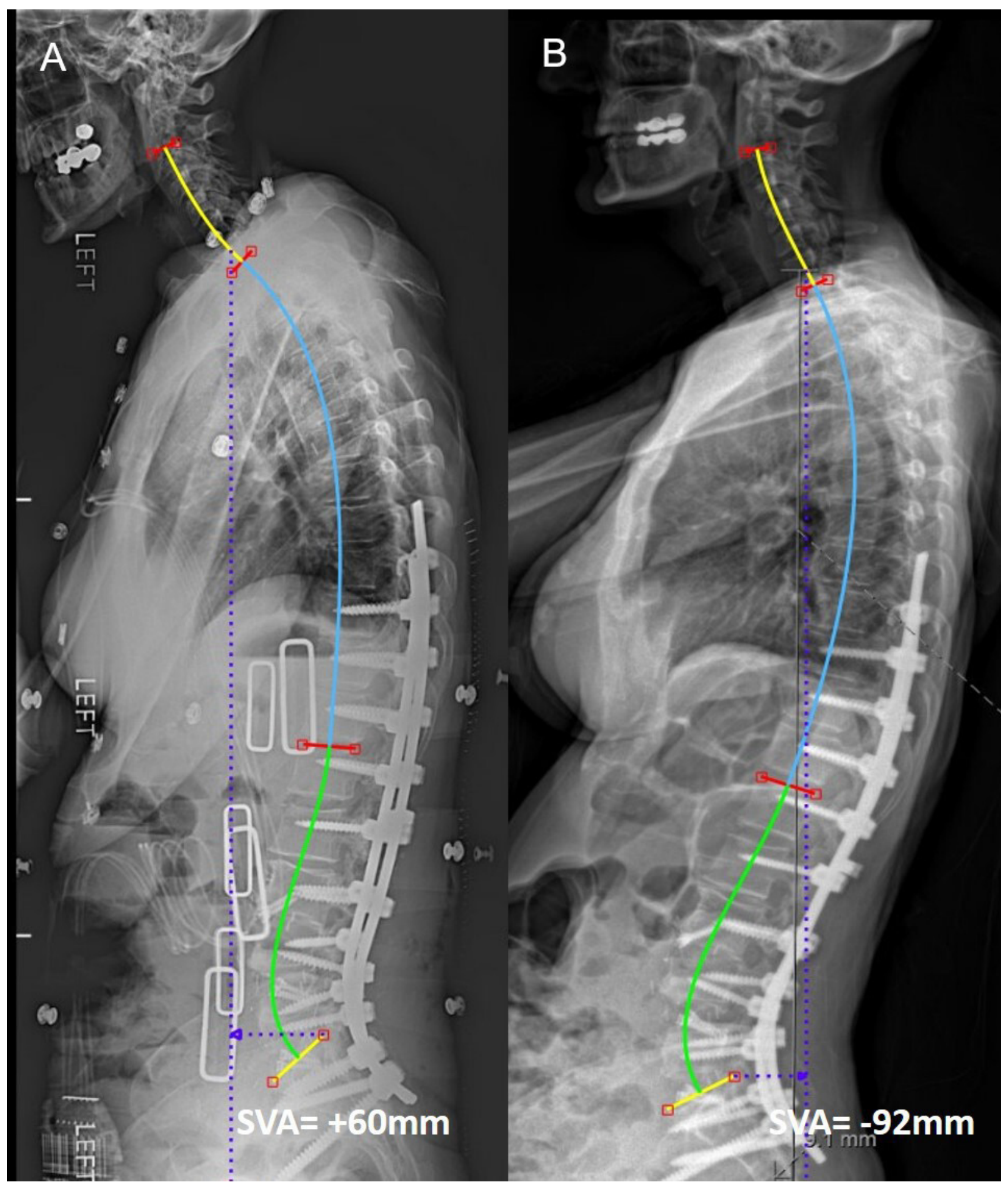

Figure 4. Lateral whole-spine radiographic scans representing changes in sagittal vertical axis (SVA) at 3-month postoperative time point compared to immediate postoperative SVA. (A) Immediate postoperative radiograph showing SVA measurement, $+60 \mathrm{~mm}$. (B) Three-month postoperative radiographs showing SVA measurement, $-32 \mathrm{~mm}$. The difference between measurements is $-92 \mathrm{~mm}$.

the paucity of previous reports on the natural course of spinal alignment changes postoperatively, we anticipate additional studies of larger size and longer follow-up periods to shed light on these variabilities and determine if they have any repercussions on the long-term outcomes of these patients.

\section{CONCLUSIONS}

Despite rigid fixation, our data suggest that significant changes may occur in the postoperative period following corrective surgery for ASD. In our study, we found a statistically significant increase in TK in the 


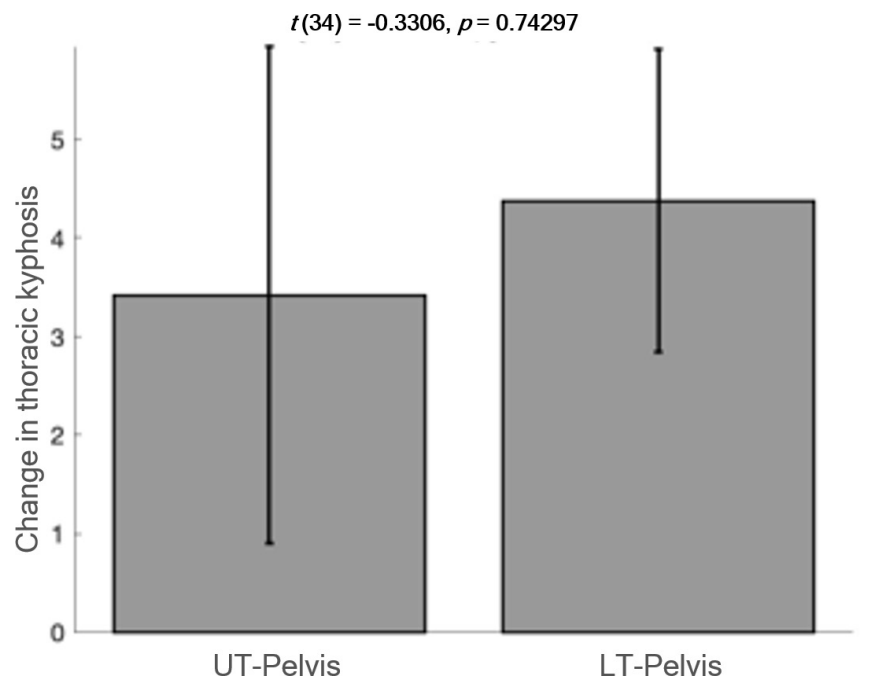

Figure 5. Bar plot representing a comparison between changes of thoracic kyphosis in upper thoracic (UT) to pelvis fusions and lower thoracic (LT) to pelvis fusions. The difference is not statically significant $(P=0.74)$.

3-month postoperative period while SVA varied greatly and, thus, we believe conclusions about the degree of correction following the ASD surgery should be reserved until after the acute postoperative period.

\section{REFERENCES}

1. Bridwell KH, Glassman S, Horton W, et al. Does treatment (nonoperative and operative) improve the two-year quality of life in patients with adult symptomatic lumbar scoliosis. Spine. 2009;34(20):2171-2178. doi:10.1097/BRS.0b013e3181a8fdc8.

2. Smith JS, Shaffrey CI, Bess S, et al. Recent and emerging advances in spinal deformity. Clin Neurosurg. 2017;80(3S):S70-S85. doi:10.1093/neuros/nyw048.

3. Youssef JA, Orndorff DO, Patty CA, et al. Current status of adult spinal deformity. Global Spine J. 2013;3(1):51-62. doi:10.105 5/s-0032-1326950.

4. Sing DC, Berven SH, Burch S, Metz LN. Increase in spinal deformity surgery in patients age 60 and older is not associated with increased complications. Spine J. 2017;17(5):627-635. doi:10.1016/j.spinee.2016.11.005.

5. Diebo BG, Shah NV, Boachie-Adjei O, et al. Adult spinal deformity. Lancet. 2019;394(10193):160-172. doi:10.1016/S01406736(19)31125-0.

6. Lutz W, Sanderson W, Scherbov S. The coming acceleration of global population ageing. Nature. 2008;451(7179):716-719. doi:10.1038/nature06516.

7. Schwab F, Patel A, Ungar B, Farcy JP, Lafage V. Adult spinal deformity-postoperative standing imbalance: how much can you tolerate? An overview of key parameters in assessing alignment and planning corrective surgery. Spine. 2010;35(25):2224-2231. doi:10.1097/BRS.0b013e3181 ee6bd4.

8. Celestre PC, Dimar JR, Glassman SD. Spinopelvic parameters: lumbar lordosis, pelvic incidence, pelvic tilt, and sacral slope: what does a spine surgeon need to know to plan a lumbar deformity correction? Neurosurg Clin N Am. 2018;29(3):323-329. doi:10.1016/j.nec.2018.03.003.
9. Arlet V, Aebi M. Junctional spinal disorders in operated adult spinal deformities: present understanding and future perspectives. Eur Spine J. 2013;22 Suppl 2:S276-95. doi:10.1007/s00586-0132676-x.

10. Scheer JK, Lafage R, Schwab FJ, et al. Under correction of sagittal deformities based on age-adjusted alignment thresholds leads to worse health-related quality of life whereas over correction provides no additional benefit. Spine. 2018;43(6):388-393. doi:10.1097/BRS.0000000000002435.

11. Board D, Yoganandan N. Biomechanics of the Aging Spine. In: Technical Paical Papers of ISA. Springer-Verlag; 2006:1-6.

12. Lee J-H, Kim K-T, Lee S-H, et al. Overcorrection of lumbar lordosis for adult spinal deformity with sagittal imbalance: comparison of radiographic outcomes between overcorrection and undercorrection. Eur Spine J. 2016;25(8):2668-2675. doi:10.1007/ s00586-016-4441-4.

13. Sebaaly A, Riouallon G, Obeid I, et al. Proximal junctional kyphosis in adult scoliosis: comparison of four radiological predictor models. Eur Spine J. 2018;27(3):613-621. doi:10.1007/s00586017-5172-x.

14. Schwab F, Lafage V, Farcy J-. P, et al. Surgical rates and operative outcome analysis in thoracolumbar and lumbar major adult scoliosis: application of the new adult deformity classification. Spine. 2007;32(24):2723-2730. doi:10.1097/BRS.0b013e31815a58f2.

15. Langella F, Villafañe JH, Damilano M, et al. Predictive accuracy of surgimap surgical planning for sagittal imbalance. Spine. 2017;42(22):E1297-E1304. doi:10.1097/BRS.0000000000002230.

16. Akbar M, Terran J, Ames CP, Lafage V, Schwab F. Use of Surgimap Spine in sagittal plane analysis, osteotomy planning, and correction calculation. Neurosurg Clin N Am. 2013;24(2):163-172. doi:10.1016/j.nec.2012.12.007.

17. Bruno AG, Anderson DE, D'Agostino J, Bouxsein ML. The effect of thoracic kyphosis and sagittal plane alignment on vertebral compressive loading. J Bone Miner Res. 2012;27(10):2144-2151. doi:10.1002/jbmr.1658.

18. Yasuda T, Hasegawa T, Yamato Y, et al. Postoperative change of thoracic kyphosis after corrective surgery for adult spinal deformity. Spine Surg Relat Res. 2018;2(4):283-289. doi:10.22603/ ssrr.2017-0081.

19. Kim HJ, Yang JH, Chang D-G, et al. Adult spinal deformity: current concepts and decision-making strategies for management. Asian Spine J. 2020;14(6):886-897. doi:10.31616/asj.2020.0568.

20. Bess S, Protopsaltis TS, Lafage V, et al. Clinical and radiographic evaluation of adult spinal deformity. $J$ Spinal Disord Tech. 2016;29(1):6-16. doi:10.1097/BSD.0000000000000352.

21. Boddu C, Jacobson M, Curtin PB, Lawlor CE, Wood K, Grottkau BE. Postoperative radiographic evaluation following adult spine deformity correction: the impact on subsequent management and associated risk of radiation exposure. Spine Deform. 2019;7(1):146-151. doi:10.1016/j.jspd.2018.05.017.

Funding: The author(s) received no financial support for the research, authorship, and/or publication of this article.

Declaration of Conflicting Interests: The author(s) declared no potential conflicts of interest with respect to the research, authorship, and/or publication of this article. 
Corresponding Author: Ahmed Albayar, Department of Neurosurgery, Hospital of University of Pennsylvania, Pennsylvania, Philadelphia, PA 19104, USA; ahmed.albayar@pennmedicine.upenn.edu

Published 24 December 2021
This manuscript is generously published free of charge by ISASS, the International Society for the Advancement of Spine Surgery. Copyright (C) 2022 ISASS. To see more or order reprints or permissions, see http:// ijssurgery.com. 